Rogier Q. Hintzen, MD, $\mathrm{PhD}$

Russell C. Dale, MD

Rinze F. Neuteboom, $\mathrm{MD}, \mathrm{PhD}$

Soe Mar, MD

Brenda Banwell, MD

Correspondence to

Dr. Hintzen:

r.hintzen@erasmusmc.nl

\title{
Pediatric acquired CNS demyelinating syndromes
}

\author{
Features associated with multiple sclerosis
}

\section{ABSTRACT}

Approximately one-third of children with an acquired demyelinating syndrome (ADS) will be diagnosed with multiple sclerosis (MS), either at onset according to the $2010 \mathrm{McD}$ conald criteria, or on the basis of clinical or MRI evidence of relapsing disease, in the majority of patients within 2-4 years. ADS in adolescents, female patients, and patients with polyfocal deficits is associated with the highest likelihood of MS, while children with acute disseminated encephalomyelitis, those with documented preceding infection, and ADS presentation in young children more commonly portends a monophasic outcome. While pediatric MS associates with similar genetic risk alleles as have been documented in adult-onset MS, such associations are not diagnostically valuable at the individual level. The presence of antibodies directed against aquaporin-4 strongly supports a diagnosis of neuromyelitis optica, and should be assayed in children manifesting with severe optic neuritis, longitudinally extensive myelitis, or brainstem/hypothalamic syndromes. Further research will determine whether other antibody signatures are indicative of relapsing demyelination distinct from MS. Neurology ${ }^{\circledR}$ 2016;87 (Suppl 2):S67-S73

\section{GLOSSARY}

ADEM = acute disseminated encephalomyelitis; $\mathbf{A D S}=$ acquired demyelinating syndromes; $\mathbf{C l}=\mathbf{c o n f i d e n c e ~ i n t e r v a l ; ~} \mathbf{C I S}=$ clinically isolated syndrome; HLA = human leukocyte antigen; HR = hazard ratio; MOG = myelin oligodendrocyte glycoprotein; $\mathbf{M S}=$ multiple sclerosis; $\mathbf{N M O}=$ neuromyelitis optica; $\mathbf{N P V}=$ negative predictive value; $\mathbf{O C B}=$ oligoclonal band; $\mathbf{O N}=$ optic neuritis; $\mathbf{O R}=$ odds ratio; $\mathbf{P P V}=$ positive predictive value; $\mathbf{S N P}=$ single nucleotide polymorphism; $\mathbf{T M}=$ transverse myelitis.

We describe the clinical presentations of acquired CNS demyelinating syndromes (ADS) in the pediatric age group, with a special focus on the clinical, MRI, laboratory, and genetic factors that identify children for whom incident ADS represents the first attack of multiple sclerosis (MS).

CLINICAL MANIFESTATIONS OF ADS IN CHILDREN Clinical presentations of acute-onset neurologic deficits associated with evidence of CNS demyelination are termed ADS. ADS phenotypes are currently classified based on clinical localization of symptoms and signs. Neuroimaging findings are supportive. The various ADS phenotypes may occur as monophasic illnesses or represent the sentinel clinical attack of a chronic demyelinating disease, such as MS. The term clinically isolated syndrome (CIS) is also often applied (and is used almost exclusively in adults presenting with an incident demyelinating attack) with specific ADS presentations such as optic neuritis (ON), transverse myelitis (TM), as well as other ADS presentations that localize to one (monofocal) or multiple (polyfocal) CNS locations ${ }^{1-6}$; however, CIS generally does not include acute disseminated encephalomyelitis (ADEM), whereas ADS encompasses both CIS and ADEM.

ON is characterized by unilateral or bilateral visual loss of variable severity, pain with ocular movement, central scotoma or reduced visual fields, reduced perception of color, and on imaging often by increased signal, swelling, and occasionally by enhancement of the optic nerves. TM manifests with acute symptoms (worsening over 4 hours to 21 days) of spinal cord dysfunction, with acute infection, postradiation, and spinal cord compression having been excluded. ${ }^{7}$ Most children with TM have a partial myelitis with bilateral motor deficits, sensory level, and depending on localization impairment, in bowel and bladder control.

From the Departments of Neurology (R.Q.H., R.F.N.) and Immunology (R.Q.H.), MS Centre ErasMS, Neurology, Erasmus MC, Rotterdam, the Netherlands; Institute for Neuroscience and Muscle Research (R.C.D.), The Kids Research Institute at the Children's Hospital at Westmead, Sydney Medical School, University of Sydney, Australia; Departments of Pediatric and Developmental Neurology (S.M.), Washington University School of Medicine, St. Louis, MO; and Division of Neurology (B.B.), The Children's Hospital of Philadelphia, Perelman School of Medicine, University of Pennsylvania.

Go to Neurology.org for full disclosures. Funding information and disclosures deemed relevant by the authors, if any, are provided at the end of the article. 
Other clinically monofocal ADS presentations include those with brainstem (i.e., internuclear ophthalmoplegia) or cerebellar localization (i.e., ataxia, dysmetria), or may manifest as focal motor/sensory deficits due to a single supratentorial CNS lesion. ${ }^{6}$

ADEM, a presentation most commonly experienced by younger children, is defined by encephalopathy (impairment in consciousness or marked irritability not accounted for by fever, recent seizure, or systemic illness) and polyfocal neurologic deficits. ${ }^{8}$ The requirement of encephalopathy is critical, as this appears to best distinguish ADEM from other ADS phenotypes.

Polyfocal deficits (neurologic signs and symptoms that require more than one CNS site for localization) without encephalopathy may also occur, and are more likely to represent the first attack of MS.

CLINICAL PARAMETERS THAT HELP DIFFERENTIATE MONOPHASIC ADS FROM MS A number of studies have tried to characterize clinical features that reliably identify those children for whom ADS represents the first attack of MS. The relative proportion of children with MS in various ADS cohorts differs across different world regions (table 1). These regional differences explain the broad range $(15 \%-$ $57 \%$ ) of MS outcomes reported in various pediatric ADS cohorts. ${ }^{1}$ Combining results from the studies, and excluding ADS studies with the most discrepant MS outcome percentages, it appears that approximately $21 \%-35 \%$ of children will be diagnosed with MS in the first 2-4 years after ADS.

The following cohort studies describe the proportion of patients with a first episode of demyelination who have MS, and the clinical features that are suggestive of MS. Logistic regression is here described as hazard ratio (HR) plus confidence intervals (CIs). If no logistic regression was performed, statistically significant differences between MS and non-MS are presented instead. However, it should be noted that many of these cohorts were analyzed before consensus definitions of pediatric MS were proposed and later revised. ${ }^{5,8}$ In addition, some cohorts were described before the testing of anti-aquaporin- 4 antibody became available.

In a prospective cohort study of 302 Canadian children with a first episode of CNS demyelination, Banwell et al. ${ }^{4}$ found that $21 \%$ were confirmed to have MS after a median of 3.1 years follow-up. Using univariate analysis, polyfocal signs without encephalopathy $(2.34,1.39-3.95)$, female sex $(1.87,1.1$ $3.1)$, and older age $(1.18,1.1-1.27)$ were predictive of MS, whereas children manifesting with ADEM were unlikely to have MS $(0.23,0.08-0.65)$.

In a nationwide retrospective follow-up study of 296 French children with a first episode of CNS demyelination, Mikaeloff et al. $^{2}$ found that after a mean follow-up of 2.9 years, 168/296 (57\%) were confirmed to have MS. A diagnosis of ADEM was against a second attack using univariate analysis (HR 0.48 , CI $0.32-0.7$ ). Using multivariate analysis, age at onset $>10$ years (HR 1.67, 1.04-2.67) was predictive of MS, and myelitis at onset (HR 0.23, 0.1$0.56)$ and severe mental status change (HR 0.59 , 0.33-1.07) were against a diagnosis of MS.

In a single-center retrospective follow-up cohort study of 118 US children with ADS, Peche et al. ${ }^{9}$ found that after a mean follow-up of 6.6 years, $25 \%$ had been diagnosed with MS. Although ADEM and younger age were against a diagnosis of MS using univariate analysis, multivariate analyses were not significant. Multivariate analysis found that brainstem and hemispheric dysfunction (as a CIS) (HR 24.6, 3.0-196) and female sex (HR 12.44, 1.03-149) were predictive of an MS diagnosis. Radiologic features were more predictive than clinical features.

In a nationwide retrospective multicenter cohort of 117 Dutch children with ADS, Neuteboom et al. ${ }^{3}$ found that after a mean follow-up of 4.5 years, $32 \%$ had been diagnosed with MS. Older age was predictive of MS, whereas encephalopathy, headache, fever, seizures, and preceding infection were against a diagnosis of MS.

In a retrospective follow-up cohort of 88 Australian children from a single center who were followed for a minimum of 1 year (mean 5.2 years), Tantsis et al. ${ }^{1}$ found that only $15 \%$ of patients had MS. Using univariate analysis, cerebellar signs were associated with MS (4.3, CI 1.35-13.74), and preceding infection was against a diagnosis of MS (0.23, CI 0.070.75). However, when applying multivariate analysis, only preceding infection remained significant (HR $0.16,0.03-0.86$ ), whereas the other predictive features of MS were radiologic.

Acute disseminated encephalomyelitis. While ADEM is typically a monophasic illness, an ADEM-like first attack can rarely represent the first attack of MS. The largest dedicated study regarding the risk of relapse after an episode of ADEM was from the French KIDSEP cohort, and described 132 children followed for a mean of 5.4 years. ${ }^{10}$ This study found that $24 / 132(18 \%)$ patients had a second attack, and 11/132 (8\%) had a third attack during the follow-up period. Using the 2012 consensus definitions for MS, 22/132 (17\%) had an ADEM event followed by one or more non-ADEM events. However, it is not clear how many of these children had dissemination in space on MRI, which is a requirement to fulfil the criteria for MS.

In the prospective nationwide Canadian study, 77 children fulfilled criteria for ADEM, and only 4/77 


\begin{tabular}{|c|c|c|c|c|c|c|c|}
\hline \multicolumn{8}{|c|}{$\begin{array}{l}\text { The proportion of patients with multiple sclerosis (MS), and clinical features for or against MS, in cohorts of children with a first episode of CNS demyelination, acute disseminated } \\
\text { encephalomyelitis (ADEM), transverse myelitis (TM), or optic neuritis (ON) }\end{array}$} \\
\hline Syndrome & Country & No. & Follow-up, y & MS, n (\%) & Features for MS/relapsing disease & Features against MS/relapsing disease & Reference \\
\hline \multirow{5}{*}{$\begin{array}{l}\text { First episode of CNS } \\
\text { demyelination (any) }\end{array}$} & Canada & 302 & 3.1 (median) & 63/302 (21) & Polyfocal signs; female sex; older age & Encephalopathy (ADEM) & Banwell et al. ${ }^{4}$ \\
\hline & France & 296 & 2.9 (mean) & $168 / 296(57)$ & Older age & Myelitis; encephalopathy & Mikaeloff et al. ${ }^{2}$ \\
\hline & United States & $118^{\mathrm{a}}$ & 6.6 (mean) & 30/118 (25) & $\begin{array}{l}\text { Brainstem or hemispheric dysfunction; } \\
\text { female sex }\end{array}$ & - & Peche et al. ${ }^{9}$ \\
\hline & Netherlands & 117 & 4.5 (mean) & $37 / 117$ (32) & Older age & $\begin{array}{l}\text { Encephalopathy; headache, fever, seizures, } \\
\text { preceding infection }\end{array}$ & Neuteboom et al. ${ }^{3}$ \\
\hline & Australia & 88 & 5.2 (mean) & $13 / 88(15)$ & Cerebellar signs & Preceding infection & Tantsis et al. ${ }^{1}$ \\
\hline \multirow[t]{3}{*}{ First episode ADEM } & France & 132 & 5.4 (mean) & 22/132 (17) & $\begin{array}{l}\text { Familial history of inflammatory demyelination; } \\
\text { ON; no sequelae after first event }\end{array}$ & - & Mikaeloff et al. ${ }^{10}$ \\
\hline & Argentina & 84 & 6.6 (mean) & $0 / 84(0)$ & - & - & Tenembaum et al. ${ }^{11}$ \\
\hline & Canada $^{\mathrm{b}}$ & 77 & 2.82 (mean) & $4 / 77$ (5) & - & Young age ( $\leq 11.85 \mathrm{y})$ & Banwell et al. ${ }^{4}$ \\
\hline First episode TM & $\begin{array}{l}\text { France, United } \\
\text { Kingdom }^{c}\end{array}$ & 95 & 1.4 (median) & $13 / 95(14)$ & Female sex & Time to nadir $\leq 24 \mathrm{~h}$ & Deiva et al. ${ }^{12}$ \\
\hline \multirow[t]{4}{*}{ First episode ON } & United States & $79^{d}$ & 19.4 (median) & $15 / 79$ (19) & Bilateral sequential or recurrent ON & Infection 2 wk before ON & Luccinetti et al. ${ }^{13}$ \\
\hline & Canada & 36 & 2.4 (mean) & $13 / 36(36)$ & $\begin{array}{l}\text { Bilateral vs unilateral ON; presence of other } \\
\text { neurologic features }\end{array}$ & - & Wilejto et al..$^{14}$ \\
\hline & United Kingdom & 44 & 1.8 (mean) & $11 / 44(25)$ & - & - & Absoud et al. ${ }^{15}$ \\
\hline & $\begin{array}{l}\text { Germany, Switzerland, } \\
\text { Austria }\end{array}$ & 357 & 4.0 (median) & $145 / 357(41)$ & Older age & - & Heussinger et al. ${ }^{16}$ \\
\hline
\end{tabular}

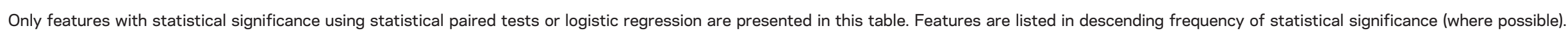
a A total of 123 in cohort, 118 with available follow-up.

${ }^{b}$ Part of a first episode of acute demyelination cohort rather than a specific ADEM cohort.

${ }^{c}$ Logistic regression odds ratio was for relapsing disease, and included neuromyelitis optica $(n=3)$ as well as MS $(n=13)$.

${ }^{\mathrm{d}}$ Ninety-five in cohort, but 79 with adequate follow-up. 
(5\%) of these children with ADEM had a diagnosis of MS after a mean of 2.82 years of follow-up. ${ }^{4}$

In a large single-center study of 84 consecutive children with ADEM, although 8/84 (10\%) had a relapsing course (referred to as biphasic disseminated encephalomyelitis), despite a mean 8.2-year follow-up (range 3-16), these 8 patients had no further clinical relapses and no new MRI lesions. ${ }^{11}$ This cohort reminds the clinician that not all relapses after ADEM represent MS.

Transverse myelitis. The likelihood of children manifesting with TM as the first attack of MS has been examined in several cohorts. ${ }^{12}$ The cohorts, ranging in size from 14 to 95 cases, generally describe a low proportion of patients who have MS (0\%-14\%), although the mean follow-up of these studies ranges from 0.5 to 8 years. The largest cohort (French and UK collaborative) describing 95 pediatric patients with TM found that $14 \%$ relapsed with a diagnosis of MS and 3\% with neuromyelitis optica (NMO), with a median follow-up time of 1.4 years (range 1-8 years) (table 1). Female sex was the strongest clinical predictor of relapsing disease (odds ratio [OR] 3.2), although notably an abnormal cerebral MRI had a significantly higher predictive value (OR 14). ${ }^{12}$ It should be noted that this study grouped the relapsing diseases (NMO and MS) together for the logistic regression. A short time to nadir of symptoms ( $<24$ hours) was against a relapsing course, and more typically seen in monophasic TM.

Optic neuritis. Lucchinetti et al. ${ }^{13}$ performed a long follow-up of childhood ON from a single US center, and found that $19 \%$ of 79 patients been diagnosed with MS after 20 years of follow-up. Of note, MRI scans were not routinely performed and testing for anti-aquaporin- 4 was not yet available. At least 4 patients in the cohort had NMO by clinical criteria. A study of 36 Canadian children with ON (unilateral in $58 \%$, bilateral in $42 \%$ ) (table 1) found that $36 \%$ of children were diagnosed with MS after a mean of 2.4 years. ${ }^{14}$ In a study of 44 British children with ON followed for a mean of 1.8 years, $25 \%$ were diagnosed with MS, but no clinical parameters were predictive of MS at first episode. ${ }^{15}$ In an important recent German-speaking retrospective multicenter cohort of 357 children with $\mathrm{ON}$ followed for a median of 4.0 years, the strongest predictors were the presence of CSF oligoclonal bands and abnormal cranial MRI, whereas features such as sex or laterality (unilateral vs bilaterality) were not predictive of MS. ${ }^{16}$

Table 1 demonstrates features that are predictive of MS (older age, female sex, polyfocal signs). In children with ADS, those presenting with ADEM and longitudinally extensive myelitis and patients younger than 12 years are less likely to be manifesting with the first attack of MS and are more likely to have a monophasic illness.

MRI ABNORMALITIES ASSOCIATED WITH MS The MRI features incorporated into the diagnostic criteria for MS and NMO are discussed in detail in "MRI in the evaluation of pediatric multiple sclerosis" (p. S88).

MRI features that assist in distinguishing children presenting with the first attack of MS from those children likely to experience a monophasic demyelinating course have been evaluated in several pediatric ADS cohorts (table 2). The French KIDMUS study showed that the presence of lesions perpendicular to the long axis of the corpus callosum and the sole presence of well-defined lesions had a sensitivity of $21 \%$, specificity of $100 \%$, positive predictive value (PPV) of $100 \%$, and negative predictive value (NPV) of $61 \%$ for identification of MS. ${ }^{17}$ The Callen et al. ${ }^{18} \mathrm{MS}$ vs ADEM criteria demonstrated that the presence of 2 out of 3 of (1) absence of diffuse bilateral lesion pattern, (2) presence of black holes, or (3) presence of 2 or more lesions with periventricular lesions yielded a sensitivity of $81 \%$, specificity of $95 \%$, PPV of $95 \%$, and NPV of $79 \%$ of identifying a child as having MS rather than ADEM. Verhey et al. ${ }^{19}$ demonstrated that the presence of $\geq 1$ periventricular T2 bright lesion and $\geq 1 \mathrm{~T} 1$-hypointense lesion had a sensitivity of $84 \%$, specificity of $93 \%$, PPV of $76 \%$, and NPV of $96 \%$ of identifying children with MS as compared to children with monophasic ADS.

When evaluating MRI features associated with monophasic $\mathrm{ON}$, as compared to those features indicative of MS or NMO, the presence of $\geq 1$ brain T2 hyperintense lesion was a strong predictor for development of MS, ${ }^{15}$ while the coexistence (or subsequent development) of longitudinally extensive myelitis or diencephalic lesions should prompt consideration of NMO. A normal brain MRI at the time of ON conveys a very low likelihood of MS, at least as evidenced by studies with up to 7 years observation. ${ }^{14,20}$ A normal brain MRI at the time of presentation with TM also conveys a low likelihood of MS. ${ }^{19}$

LABORATORY PARAMETERS ASSOCIATED WITH MS CSF analysis is an important tool in the diagnostic workup of childhood ADS. Although specific CSF findings are not obligatory for MS diagnosis, CSF is informative on MS likelihood and can play a key role in prompting evaluation of other etiologies.

The CSF cell count and composition should first be evaluated. In pediatric MS, CSF pleocytosis (predominantly monocytic) has been described in $52 \%-66 \%$ of patients. $^{21-23}$ A cell count above 60 cells $/ \mu \mathrm{L}$ is rare in pediatric MS. ${ }^{21}$ CSF neutrophils 


\begin{tabular}{|c|c|c|c|}
\hline \multirow[t]{2}{*}{ Table 2} & \multicolumn{3}{|c|}{$\begin{array}{l}\text { MRI features that assist in distinguishing children presenting with the first attack of multiple } \\
\text { sclerosis (MS) }\end{array}$} \\
\hline & KIDMUS $^{15}$ & Callen et al. ${ }^{18}$ MS vs ADEM & Verhey et al. ${ }^{19}$ \\
\hline Criteria & $\begin{array}{l}\text { Lesions perpendicular to the long } \\
\text { axis of the corpus callosum; the sole } \\
\text { presence of well-defined lesions }\end{array}$ & $\begin{array}{l}\text { Two out of three of (1) absence of diffuse } \\
\text { bilateral lesion pattern; (2) presence of black } \\
\text { holes; ( } 3 \text { ) presence of } 2 \text { or more lesions with } \\
\text { periventricular lesions }\end{array}$ & $\begin{array}{l}\geq 1 \text { Periventricular T2 } \\
\text { bright lesion; } \geq 1 \\
\text { hypointense lesion }\end{array}$ \\
\hline Sensitivity, \% & 21 & 81 & 84 \\
\hline Specificity, \% & 100 & 95 & 93 \\
\hline PPV, \% & 100 & 95 & 76 \\
\hline NPV, \% & 61 & 79 & 96 \\
\hline
\end{tabular}

Abbreviations: $\mathrm{ADEM}$ = acute disseminated encephalomyelitis; NPV = negative predictive value; $\mathrm{PPV}$ = positive predictive value.

should prompt consideration of infection, and cellular atypia a search for malignancy.

The presence of oligoclonal bands (OCBs) in CSF that are absent in serum is strongly supportive of an MS diagnosis and can be detected in up to $90 \%$ of patients using isoelectric focusing technique in an experienced laboratory. ${ }^{3,4,21-23}$ Two studies have investigated the presence of CSF OCBs in children with MS aged under and above 11 years; CSF OCBs were detected in $43 \%-60 \%$ of the younger group and $63 \%-73 \%$ in the children older than $11 .^{22,23}$ Detection of CSF OCBs increases when analyzed CSF is obtained serially. ${ }^{23}$ The presence in CSF of OCBs is not pathognomonic for MS, as OCBs are detected in $8 \%-15 \%$ of children with ADS who have not been subsequently diagnosed with MS. ${ }^{4,23}$ CSF OCBs have recently been shown to have useful predictive potential: in a cohort of 357 children with ON, intrathecal OCBs were strongly predictive of MS (HR 3.69, 95\% CI 2.32-5.86, $p<0.001) .{ }^{16}$

SERUM ANTIBODIES TO MYELIN OLIGODENDROCYTE GLYCOPROTEIN (MOG) Patients with ON, myelitis, and brainstem syndromes should have testing for AQP4 antibodies. MOG antibodies may also have a role in disease classifications, although this has not been incorporated into consensus definitions to date. There are emerging data to suggest that MOG antibodies define a non-MS subgroup of CNS demyelination, and are found frequently in patients with $\mathrm{ADEM}, \mathrm{ON}$, and myelitis (see "Immunopathophysiology of pediatric CNS inflammatory demyelinating disease,” p. S12).

Several studies have focused on the antibody response to MOG in pediatric $\mathrm{ADS}$ cohorts. ${ }^{24-26}$ The percentage of anti-MOG antibody-positive patients within these ADS groups ranges between $12 \%$ and $35 \%$. Children with antibodies to MOG are typically younger than children without antibodies to MOG. A Dutch nationwide cohort study on 117 children with ADS showed that patients with ADS with antibodies to MOG more frequently present with a polyfocal disease onset with or without encephalopathy, next to TM (recurrent), ON, or NMO negative for antibodies to aquaporin-4. ${ }^{24}$ Children with multiphasic ADS not fulfilling MS diagnostic criteria were likely to be positive (8/11) for anti-MOG antibodies. These children with a multiphasic disease course often have ADEM followed by one or more ON. ${ }^{24,26}$ Similar results have been documented by other groups, ${ }^{25}$ indicating that the presence of anti-MOG positivity tested with cell-based assays statistically suggests against a diagnosis of MS. Still, other studies using cell-based assays have found anti-MOG antibodies particularly in children diagnosed with MS younger than 10 years, ${ }^{27}$ suggesting an age-dependent presence of MOG antibodies in pediatric MS.

GENETIC BACKGROUND ASSOCIATED WITH MS Although there are now at least 159 genes involved in genetic vulnerability (Phil de Jager, personal communication on behalf of the International MS Genetics Consortium and abstract ECTRIMS, 2014), the presence of any one of these genetic variations is not predictive of MS, nor does the absence of these genetic variations exclude MS. Therefore, there is no reason to perform genetic testing to support a diagnosis of MS in an individual patient.

Genetic factors, however, do influence MS risk, and recent studies have indicated that the genes overrepresented in MS are not similarly overrepresented in monophasic ADS. The main genetic locus associated worldwide with adult MS is within the major histocompatibility complex, and in particular risk associates with the human leukocyte antigen (HLA)DRB $1 * 15$ allele. ${ }^{28}$ In a Canadian study of pediatric ADS, the presence of 1 or 2 HLA-DRB $1 * 15$ alleles conferred an OR of 2.7 for MS outcome. ${ }^{29}$ Children with positive MOG antibodies have similar rates of HLA-DRB $1 * 15$ allele to the general population $(3 / 18)$, whereas children with negative MOG antibodies have higher rates of HLA-DRB1*15 allele 
(7/22), further strengthening the case that MOG antibody supports a non-MS diagnosis, although larger cohorts are required to further test this association. ${ }^{30}$

Another study evaluated the frequencies of 57 of the newly identified non-HLA MS risk single nucleotide polymorphisms (SNPs) in 188 children with ADS, of whom 53 individuals had a subsequent diagnosis of MS. ${ }^{31}$ In order to control the effect of genetic variation due to ancestry, only patients with European ancestry were included. Control groups were $456 \mathrm{pa}-$ tients with adult MS and 2,046 healthy adults. A weighted genetic risk score of this set of SNPs discriminated between children with MS vs those with monophasic ADS. The association with MS further increased with the addition of (female) sex and HLADRB1*15. Interestingly, the combined effect of the 57 SNPs exceeded the effect of the HLA DRB $1 * 15$ alone.

DISCUSSION The prompt diagnosis of MS in children and adolescents is aided by recognition of the various clinical manifestations, by specific lesion patterns on baseline and serial MRI, and by the presence of intrathecal OCBs. No pattern of serum antibodies or immune cell signatures has yet been identified that reliably identifies children with MS, but antibodies against aquaporin- 4 and myelin oligodendroglial protein serve to identify children who are likely to have diseases distinct from MS.

\section{AUTHOR CONTRIBUTIONS}

Rogier Q. Hintzen: abstract, genetic background, editing, concluding remarks. Russell C. Dale: clinical parameters, abstract. Rinze F. Neuteboom: laboratory parameters, serum antibodies. Soe Mar: MRI abnormalities. Brenda Banwell: clinical manifestations of ADS, editing.

\section{STUDY FUNDING}

This supplement is made possible by funding from the MS Cure Fund, Danish MS Society, German MS Society, Italian MS Association, MS International Federation, MS Research Foundation (Netherlands), National MS Society (USA) and Swiss MS Society.

\section{DISCLOSURE}

R. Hintzen received honoraria for serving on advisory boards from Biogen Idec, Roche, and Sanofi. He participated in trials with Biogen Idec, Merck-Serono, Roche, Genzyme, and Novartis. He is on the editorial board of Multiple Sclerosis and Related Disorders. R. Dale has received honoraria from Biogen Idec and Bristol-Myers-Squibb. R. Neuteboom is a member of the LAREB Scientific Advisory Board. S. Mar reports no disclosures relevant to the manuscript. B. Banwell serves as a central MRI reviewer for Novartis. Dr. Banwell provides advice on clinical trial design to Novartis, Biogen Idec, Sanofi, and Teva Neuroscience. She is not remunerated for this work. Dr. Banwell is a senior editor for Multiple Sclerosis and Related Disorders. Go to Neurology.org for full disclosures.

Received August 19, 2015. Accepted in final form February 1, 2016.

\section{REFERENCES}

1. Tantsis EM, Prelog K, Brilot F, Dale RC. Risk of multiple sclerosis after a first demyelinating syndrome in an Australian paediatric cohort: clinical, radiological features and application of the McDonald 2010 MRI criteria. Mult Scler 2013;19:1749-1759.
2. Mikaeloff Y, Suissa S, Vallee L, et al. First episode of acute CNS inflammatory demyelination in childhood: prognostic factors for multiple sclerosis and disability. J Pediatr 2004; 144:246-252.

3. Neuteboom RF, Boon M, Catsman Berrevoets CE, et al. Prognostic factors after a first attack of inflammatory CNS demyelination in children. Neurology 2008;71:967-973.

4. Banwell B, Bar-Or A, Arnold DL, et al. Clinical, environmental, and genetic determinants of multiple sclerosis in children with acute demyelination: a prospective national cohort study. Lancet Neurol 2011;10:436-445.

5. Krupp LB, Banwell B, Tenembaum S. Consensus definitions proposed for pediatric multiple sclerosis and related disorders. Neurology 2007;68:S7-S12.

6. Miller DH, Chard DT, Ciccarelli O. Clinically isolated syndromes. Lancet Neurol 2012;11:157-169.

7. Transverse Myelitis Consortium Working Group. Proposed diagnostic criteria and nosology of acute transverse myelitis. Neurology 2002;59:499-505.

8. Krupp LB, Tardieu M, Amato MP, et al. International Pediatric Multiple Sclerosis Study Group criteria for pediatric multiple sclerosis and immune-mediated central nervous system demyelinating disorders: revisions to the 2007 definitions. Mult Scler 2013;19:1261-1267.

9. Peche SS, Alshekhlee A, Kelly J, Lenox J, Mar S. A long-term follow-up study using IPMSSG criteria in children with CNS demyelination. Pediatr Neurol 2013;49:329-334.

10. Mikaeloff Y, Caridade G, Husson B, Suissa S, Tardieu M. Acute disseminated encephalomyelitis cohort study: prognostic factors for relapse. Eur J Paediatr Neurol 2007;11:90-95.

11. Tenembaum S, Chamoles N, Fejerman N. Acute disseminated encephalomyelitis: a long-term follow-up study of 84 pediatric patients. Neurology 2002;59:1224-1231.

12. Deiva K, Absoud M, Hemingway C, et al. Acute idiopathic transverse myelitis in children: early predictors of relapse and disability. Neurology 2015;84:341-349.

13. Lucchinetti CF, Kiers L, O’Duffy A, et al. Risk factors for developing multiple sclerosis after childhood optic neuritis. Neurology 1997;49:1413-1418.

14. Wilejto M, Shroff M, Buncic JR, Kennedy J, Goia C, Banwell B. The clinical features, MRI findings, and outcome of optic neuritis in children. Neurology 2006;67: 258-262.

15. Absoud M, Cummins C, Desai N, et al. Childhood optic neuritis clinical features and outcome. Arch Dis Child 2011;96:860-862.

16. Heussinger N, Kontopantelis E, Gburek-Augustat J, et al; for GRACE-MS (German-speaking Research Alliance for Children with Multiple Sclerosis). Oligoclonal bands predict multiple sclerosis in children with optic neuritis. Ann Neurol 2015;77:1076-1082.

17. Mikaeloff Y, Adamsbaum C, Husson B, et al. MRI prognostic factors for relapse after acute CNS inflammatory demyelination in childhood. Brain 2004;127:1942-1947.

18. Callen DJ, Shroff MM, Branson HM, et al. Role of MRI in the differentiation of ADEM from MS in children. Neurology 2009;72:968-973.

19. Verhey LH, Branson HM, Shroff MM, et al. MRI parameters for prediction of multiple sclerosis diagnosis in children with acute CNS demyelination: a prospective national cohort study. Lancet Neurol 2011;10:1065-1073.

20. Bonhomme GR, Waldman AT, Balcer LJ, et al. Pediatric optic neuritis: brain MRI abnormalities and risk of multiple sclerosis. Neurology 2009;72:881-885. 
21. Pohl D, Rostasy K, Reiber H, Hanefeld F. CSF characteristics in early-onset multiple sclerosis. Neurology 2004;63: 1966-1967.

22. Chabas D, Ness J, Belman A, et al. Younger children with MS have a distinct CSF inflammatory profile at disease onset. Neurology 2010;74:399-405.

23. Huppke B, Ellenberger D, Rosewich H, Friede T, Gärtner J, Huppke P. Clinical presentation of pediatric multiple sclerosis before puberty. Eur J Neurol 2014;21:441-446.

24. Ketelslegers IA, Van Pelt DE, Bryde S, et al. Anti-MOG antibodies plead against MS diagnosis in an acquired demyelinating syndromes cohort. Mult Scler 2015;21: 1513-1520.

25. Hacohen Y, Absoud M, Deiva K, et al. Myelin oligodendrocyte glycoprotein antibodies are associated with a non-MS course in children. Neurol Neuroimmunol Neuroinflamm 2015;2:e81.

26. Huppke P, Rostasy K, Karenfort M, et al. Acute disseminated encephalomyelitis followed by recurrent or monophasic optic neuritis in pediatric patients. Mult Scler 2013;19:941-946.
27. McLaughlin KA, Chitnis T, Newcombe J, et al. Agedependent $\mathrm{B}$ cell autoimmunity to a myelin surface antigen in pediatric multiple sclerosis. J Immunol 2009;183: 4067-4076.

28. Sadovnick AD, Hintzen RQ. Genetics of pediatric multiple sclerosis. In: Demyelinating Disorders of the Central Nervous System in Childhood. Cambridge: Cambridge University Press; 2011:169-182.

29. Disanto G, Magalhaes S, Handel AE, et al. HLADRB1 confers increased risk of pediatric-onset MS in children with acquired demyelination. Neurology 2011;76: 781-786.

30. Dale RC, Tantsis EM, Merheb V, et al. Antibodies to MOG have a demyelination phenotype and affect oligodendrocyte cytoskeleton. Neurol Neuroimmunol Neuroinflamm 2014;1:e12.

31. van Pelt ED, Mescheriakova JY, Makhani N, et al. Risk genes associated with pediatric-onset MS but not with monophasic acquired CNS demyelination. Neurology 2013;81:1996-2001. 


\section{Neurology}

\section{Pediatric acquired CNS demyelinating syndromes: Features associated with multiple sclerosis}

Rogier Q. Hintzen, Russell C. Dale, Rinze F. Neuteboom, et al. Neurology 2016;87;:S67-S73

DOI 10.1212/WNL.0000000000002881

This information is current as of August 29, 2016

\section{Updated Information \&} Services

References

Citations

Subspecialty Collections

Permissions \& Licensing

Reprints including high resolution figures, can be found at: http://n.neurology.org/content/87/9_Supplement_2/S67.full

This article cites 30 articles, 2 of which you can access for free at: http://n.neurology.org/content/87/9_Supplement_2/S67.full\#ref-list-1

This article has been cited by 2 HighWire-hosted articles: http://n.neurology.org/content/87/9_Supplement_2/S67.full\#\#otherartic les

This article, along with others on similar topics, appears in the following collection(s):

Multiple sclerosis

http://n.neurology.org/cgi/collection/multiple_sclerosis

Information about reproducing this article in parts (figures,tables) or in its entirety can be found online at:

http://www.neurology.org/about/about_the_journal\#permissions

Information about ordering reprints can be found online:

http://n.neurology.org/subscribers/advertise

Neurology ${ }^{\circledR}$ is the official journal of the American Academy of Neurology. Published continuously since 1951, it is now a weekly with 48 issues per year. Copyright @ 2016 American Academy of Neurology. All rights reserved. Print ISSN: 0028-3878. Online ISSN: 1526-632X.

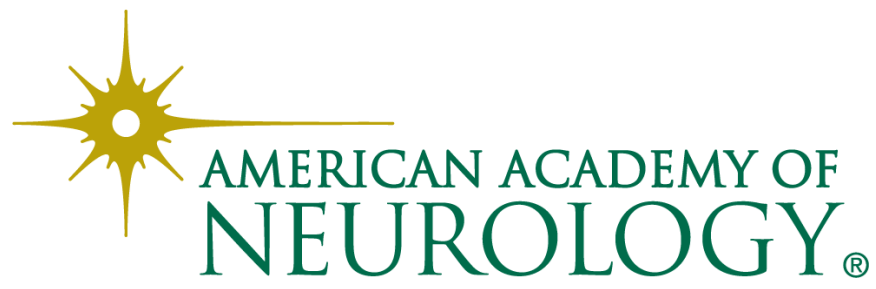

